\title{
Flavonoids from Radix Tetrastigmae improve LPS-induced acute lung injury via the TLR4/MD-2-mediated pathway
}

\author{
DAN-DAN LIU ${ }^{1,2}$, GANG CAO $^{1}$, LI-KAI HAN ${ }^{3}$, YI-LU YE ${ }^{2}$, QI ZHANG ${ }^{2}$, YU-HAN SIMA ${ }^{4}$ and WEI-HONG GE ${ }^{1}$ \\ ${ }^{1}$ Department of Pharmacy, Zhejiang Chinese Medical University; ${ }^{2}$ Department of Basic Medicine, Zhejiang Medical College, \\ Hangzhou, Zhejiang 310053; ${ }^{3}$ Pathological Department, People's Hospital of Zhejiang, Hangzhou, Zhejiang 310046; \\ ${ }^{4}$ Hangzhou Xuejun High School, Hangzhou, Zhejiang 310012, P.R. China
}

Received July 21, 2015; Accepted May 27, 2016

DOI: $10.3892 / \mathrm{mmr} .2016 .5412$

\begin{abstract}
Total flavonoids isolated from Radix Tetrastigmae (RTFs) possess immunomodulatory activity, particularly on inflammation. In mice with lipopolysaccharide (LPS)-induced acute lung injury (ALI), treatment with RTFs at 40, 80 and $160 \mathrm{mg} / \mathrm{kg}$ significantly reduced leukocyte infiltration, improved histopathological changes in lung tissues and decreased the LPS-induced production of several inflammatory mediators in the bronchoalveolar lavage fluid (BALF), which included the chemotatic factors, granulocyte colony-stimulating factor, monocyte inflammatory protein-1 $\alpha$ and B-lymphocyte colony inflammatory cytokines, including interleukin (IL)-1 $\beta$, IL-6, IL-12p40 and tumor necrosis factor- $\alpha$, in a dose-dependent manner. In addition, the expression of the Toll-like receptor 4 (TLR4)/myeloid differentiation factor-2 (MD-2) compound, the phosphorylation of p38 mitogen-activated protein kinase (p38MAPK), c-Jun N-terminal kinase (JNK) and nuclear transcription factor $-\kappa \mathrm{B}(\mathrm{NF}-\kappa \mathrm{B})$, in addition to the DNA binding activity of NF- $\mathrm{B}$ p65 in lung tissues, were all attenuated following RTF treatment. However, RTF treatment had no effect on extracellular signal-regulated kinase (ERK). In conclusion, RTFs contributed to the regulation of LPS-induced ALI through the TLR4/MD-2-mediated NF- $\mathrm{B}, \mathrm{JNK}$ and p38MAPK pathways. This may be a potential therapeutic option for the treatment of inflammatory diseases.
\end{abstract}

Correspondence to: Professor Wei-Hong Ge, Department of Pharmacy, Zhejiang Chinese Medical University, 546 Binwen Road, Hangzhou, Zhejiang 310053, P.R. China

E-mail: geweihong@hotmail.com

Abbreviations: RTFs, flavonoids isolated from Radix Tetrastigmae; ALI, acute lung injury; JNK, c-Jun N-terminal kinase; TLR4, Toll-like receptor 4; MD-2, myeloid differentiation factor-2; NF- $\kappa \mathrm{B}$, nuclear transcription factor- $\kappa \mathrm{B}$; MAPKs, mitogen-activated protein kinases; LPS, lipopolysaccharide

Key words: Radix Tetrastigmae flavonoids, Toll-like receptor 4, myeloid differentiation factor-2, anti-inflammation, c-Jun N-terminal kinase, nuclear transcription factor- $\kappa \mathrm{B}$, p38 mitogen-activated protein kinase, lipopolysaccharide

\section{Introduction}

Acute lung injury (ALI) and its severe manifestation, acute respiratory distress syndrome (ARDS), are well-known life-threatening diseases with high morbidity rates $(35-50 \%)$ in critically ill patients (1). The multiple etiologies, including severe sepsis, pneumonia, lung abscess and severe burns, cause uncontrolled and self-amplified pulmonary inflammation, which are at the center of the pathology of ALI/ARDS (2-5). Studies have been confirmed that the alveolar epithelium and pulmonary endothelium are the primary injury targets of this disease (3-6). Although substantial progress has been made in understanding the pathogenesis and pathophysiology of ALI/ARDS, the treatment remains limited.

It has been confirmed that intratracheal instillation of the endotoxin, lipopolysaccharide (LPS), can reproducibly induce ALI (7). LPS induces inflammatory cell infiltration into lung tissues, causing the release of proinflammatory cytokines, reactive oxygen species and chemotactic factors (8). These changes can cause lung edema, alveolar hemorrhage and substantial inflammation. In mammals, the Toll-like receptor 4 (TLR4)/myeloid differentiation factor-2 (MD-2) complex constitutes an essential component of the LPS receptor system (9). Common downstream signal transduction pathways of TLR4/MD-2, which have been shown to mediate inflammatory responses in lung injury, include nuclear factor- $\kappa \mathrm{B}(\mathrm{NF}-\kappa \mathrm{B})$ and mitogen-activated protein kinases (MAPKs) (10). Major signaling pathways regulating cellular growth and the responses to cytokines and stress occur through the conserved MAPK family, which consists of p42/44 extracellular signal-regulated kinase (ERK), p38 MAPK and c-Jun N-terminal kinase (JNK) (11). NF- $\kappa \mathrm{B}$ is a critical transcription factor required for maximal expression of several cytokines, and is involved in the pathogenesis of acute lung injury. Reports have demonstrated that the severity of inflammation in ALI/ARDS is markedly reduced by inhibition of the NF- $\mathrm{B}$ signaling pathway (12-15). Therefore, drugs focusing on downregulating the TLR4/MD-2-mediated MAPK and NF- $\kappa \mathrm{B}$ signaling pathways have a potent therapeutic effect for the treatment of ALI (16).

Radix Tetrastigmae (RT) is a commonly used Chinese traditional medicine. The total flavonoid compounds isolated from RT (RTFs), the root of Tetrastigma hemsleyanum Diels 
et Gilg), are the predominant active ingredients and have wide biological and pharmacological functions, including antitumor, liver-protecting, antiviral, anti-inflammatory, immunoregulatory effects and other pharmacological effects, with the exception that it is basically non-toxic (17-19). The present study investigated the effect of RTFs on mice with LPS-induced ALI for the first time, to the best of our knowledge. Elucidation of the mechanisms, TLR4/MD-2-mediated MAPK and NF- $\kappa$ B signaling pathways and associated downstream regulators were also investigated, with the aim of determining the therapeutic effects of RTFs on ALI.

\section{Materials and methods}

Flavonoid isolation. The total flavonoids were extracted from RT, according to the method previously reported (20) with mild revision. Briefly, decoction powder of RT from Guizhou (China) was extracted twice with $60 \%$ ethanol (v/w) in a boiling water bath for $90 \mathrm{~min}$, followed by filtering and concentration using reduced pressure distillation. The crude extracts were dissolved in water and purified through resin HPD826. Total flavonoid extracts were obtained with further distillation under a vacuum with lyophilization of the eluant. The quality of the total flavonoid extract was determined using colorimetric $\mathrm{HCl}-\mathrm{Mg}$ and aluminum chloride reactions, followed by high-performance liquid chromatography $(20,21)$.

Animals. Male BALB/c mice $(\mathrm{n}=60)$ were purchased from SLRC Laboratory Animal, Ltd. (Shanghai, China). These mice used in the present study were $\sim 10$-week old mice (20-25 g), matched for age and weight. The animals were maintained in room with regulated temperature $\left(22-24^{\circ} \mathrm{C}\right)$, humidity $(55 \pm 5 \%)$ and light (12 h light-dark cycle), and with access to food and water ad libitum. All procedures were performed in accordance with the Guidelines for Animal Experimentation of Zhejiang University (Zhejiang, China).

Mouse model of ALI. The mice were randomly divided into five groups ( $n=12 /$ group): Control [phosphate-buffered saline (PBS)]; model (2.5 mg/kg LPS) and TRF (40, 80 or $160 \mathrm{mg} / \mathrm{kg}+2.5 \mathrm{mg} / \mathrm{kg}$ LPS). All mice were challenged intratracheally with either $25 \mu \mathrm{l} / \mathrm{PBS}$ alone or $25 \mu \mathrm{l}$ PBS containing $25 \mu \mathrm{g}$ LPS (per $10 \mathrm{~g}$ body weight) under anesthesia with intraperitoneal injection of chloral hydrate $(400 \mathrm{mg} / \mathrm{kg})$. The mice were intragastrically administered with $100 \mu \mathrm{l}$ PBS or RTF 30 min prior to LPS challenge and for 2 days after LPS challenge. On the 4th day, mice were anesthetized with chloral hydrate (0.4 g/kg; Sigma-Aldrich; St. Louis, MO, USA) to collect the bronchoalveolar lavage fluid (BALF) and lung tissues and then sacrificed by overdose of chloral hydrate. The severity of lung injury was assessed by pathological changes in the lung tissues and cellular profiles in the BALF.

Collection of BALF and leukocyte counting. The BALF was collected, as previously described (22). Briefly, under anesthesia, the trachea was cannulated and the lungs were lavaged three times with PBS $(0.5 \mathrm{ml}$ each time). The collected BALF was centrifuged at $200 \mathrm{x}$ g for $5 \mathrm{~min}$ at $4^{\circ} \mathrm{C}$, and the supernatant was frozen at $-80^{\circ} \mathrm{C}$ for subsequent biochemical analysis. The pelleted cells were then re-suspended in PBS for cell counting. The numbers of leukocytes were enumerated using a hemocytometer. For differential counts, smears of the BALF cells $\left(\sim 10^{5}\right.$ cells $\left./ \mathrm{ml}\right)$ from each mouse were prepared by centrifugation $\left(4^{\circ} \mathrm{C}\right.$ at $200 \mathrm{x}$ g for $\left.5 \mathrm{~min}\right)$ and then stained with Wright-Giemsa solution.

Inflammatory cytokine antibody array and ELISA. The secretion of different cytokines from the BALF was measured using a semi-quantitative mouse inflammation antibody array from RayBiotech (Guangzhou, China). In brief, antibodies against 40 different inflammatory cytokines were spotted onto the cytokine array by the manufacturer. Initially, $100 \mu$ l blocking buffer was added each well and incubated at room temperature for $30 \mathrm{~min}$ to block the slides. Subsequently, $100 \mu \mathrm{l}$ sample was added to each sub-array at room temperature overnight, prior to incubation with biotin-conjugated primary antibodies. Following washing, Cy3-conjugated streptavidin was added for 2 h. Finally the chip was scanned using a GenePix 4000B microarray scanner (Molecular Devices, Sunnyvale, CA, USA). The images were analyzed using the GenePix Pro 5.0 software program (Molecular Devices), and the levels of cytokines were quantified according to the standard curve calibrated from the same array.

The secretion of interleukin (IL)-1 $\beta$, tumor necrosis factor (TNF)- $\alpha$, IL-6 and IL-12p40 in the BALF were further measured using an eBioscience ELISA kit (eBioscience, San Diego, CA, USA), according to the manufacturer's protocol. In addition, activation of the MAPK pathway in the lung tissues was also detected using ELISA kits (Elisa Biotech, Shanghai, China), including p38, phosphorylated (p)-p38, ERK, p-ERK, JNK and p-JNK.

Histopathologic examination of lung tissues. To characterize the histological alterations in the lung tissues, the lungs were excised and fixed in $10 \%$ buffered formalin. Following dehydration with graded alcohol and embedding in paraffin, the lung tissues were cut into $4 \mu \mathrm{m}$ sections, and subsequently stained with hematoxylin and eosin. The lung injury was scored by an observer in a blinded-manner under a light microscope, according to pathological changes, including cell infiltration and accumulation, pulmonary edema, hemorrhage and the thickness of the alveolar wall. Each indicator was graded according to a five-point scale: $0=$ minimal damage, $1=$ mild damage. $2=$ moderate damage, $3=$ severe damage and $4=$ maximal damage. Therefore, the severity of lung injury was assessed according to the sum of the individual indicator scores (23).

Western blot analysis of lung tissues. The lung tissues were homogenized and quantified using a BCA protein assay kit (Pierce Biotechnology, USA). Each protein sample $(20 \mu \mathrm{g})$ was mixed with the sample buffer to a final concentration of $0.06 \mathrm{M}$ Tris- $\mathrm{HCl}, \mathrm{pH} 6.8,2 \%$ SDS, $5 \% \beta$-mercaptoethanol, $10 \%$ glycerol and $0.025 \%$ bromophenol blue. Equal quantities of protein were loaded per well, separated by SDS-PAGE and transferred onto PVDF membranes (EMD Millipore, Billerica, CA, USA). Subsequent to blocking with $5 \%$ nonfat milk for $1 \mathrm{~h}$, the membranes were probed with monoclonal antibodies rabbit anti-mouse monoclonal antibodies against MD-2 (cat. no. sc-20668; Santa Cruz Biotechnology, Inc., Danvers, MA, USA), TLR4 (cat. no. DR4841 UcallM Biotechnology 


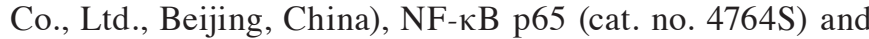
p-NF- $\kappa$ B p65 (cat. no. 3033S) (Cell Signaling Technology, Beverly, MA, USA) overnight at $4^{\circ} \mathrm{C}$. Membranes were then incubated with GAPDH-HRP mAb (LK9002T) and goat anti-rabbit secondary antibody (LK2001) (Sungene Biotech, Co., Ltd., Tianjing, China). The blots were normalized to GAPDH to correct for differences in loading of the proteins. Densitometric values of the immunoblot signals were obtained from three separate experiments using Pro 3DS 6.0 image software (National Institutes of Health, Bethesda, MA, USA).

$N F-\kappa B$ p 65 activity assay. NF- $\kappa \mathrm{B}$ activity was measured using an ELISA-based TransAM NF- $\kappa$ B kit (Active Motif, Carlsbad, CA, USA) on the nuclear protein extracts. The Nuclear Extract kit was obtained from Active Motif for the preparation of nuclear extracts from the lung tissues. The TransAM assay for $\mathrm{NF}-\kappa \mathrm{B}$ p65 activity was performed, according to the manufacturer's protocol $(13,14)$. Briefly, oligonucleotides containing the NF- $\kappa$ B consensus binding site (5'-GGGACTTCC-3') were immobilized on a 96-well plate. The active forms of $\mathrm{NF}-\kappa \mathrm{B}$ in the nuclear extracts were bound to the oligonucleotides on the plate and detected colorimetrically. The samples were measured at an absorbance of $450 \mathrm{~nm}$ on a spectrophotometer, with a reference wavelength of $650 \mathrm{~nm}$.

Statistical analysis. All values are expressed as the mean \pm standard deviation. Differences between the mean values of normally distributed data were assessed using one-way analysis of variance (Dunnett's $t$-test) and two-tailed Student's $t$-test using SPSS software v.19 (IBM, Armonk, NY, USA). $\mathrm{P}<0.05$ was considered to indicate a statistically significant difference.

\section{Results}

RTFS decreases LPS-induced leukocyte infiltration in the $B A L F$. The recruitment of inflammatory cells into pulmonary alveoli is important in ALI. Following 3 days of LPS instillation, the levels of total leukocytes, neutrophils and macrophages in the BALF were significantly increased in all groups exposed to LPS, compared with those of the control group. RTF treatment (40, 80 and $160 \mathrm{mg} / \mathrm{kg}$ ) significantly reduced LPS-induced leukocyte exudation (Fig. 1; $\mathrm{P}<0.05$ ), particularly the numbers of neutrophils and macrophages $(\mathrm{P}<0.01)$.

RTFs attenuate the secretion of inflammatory factors in $B A L F$. Elevation in the levels of proinflammatory cytokines is a typical response to LPS challenge. In the present study, the release of 40 inflammatory cytokines from the BALF of RTF-treated mice was determined using a mouse cytokine antibody array. In the microarray, any $\geq 1.5$-fold increase or $\leq 0.65$-fold decrease in signal intensity for a single analyte between samples or groups was considered a measurable and significant difference in expression, provided that the sets of signals were well above background (mean background \pm two standard deviations; accuracy $\sim 95 \%$ ). Compared with the model, the release of B lymphocyte chemoattractant (BLC), granulocyte colony stimulating factor (GCSF), IL-1 $\beta$, IL-6, IL-12p40/p70, macrophage inflammatory protein-1 $\alpha$ (MIP-1 $\alpha$ ), tissue inhibitor of metalloproteinase-1 (TIMP-1)

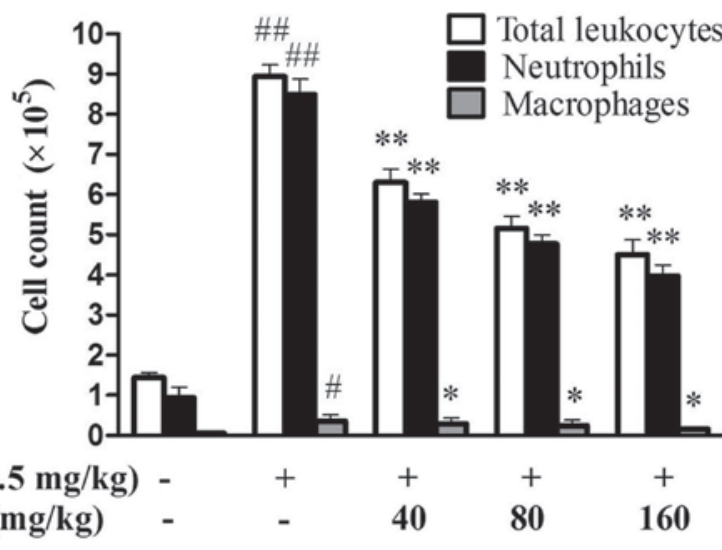

Figure 1. RTFs inhibit inflammatory cell infiltration in mice with LPS-induced acute lung injury. BALF was collected following treatment with LPS $(2.5 \mathrm{mg} / \mathrm{kg})$ and/or RTFs (40, 80 and $160 \mathrm{mg} / \mathrm{kg}$ ) for 3 days, and the numbers of total leukocytes, neutrophils and macrophages in the BALF were examined. The data are expressed as the mean \pm standard deviation of 10-12 mice/group. ${ }^{\#} \mathrm{P}<0.05$ and ${ }^{\# \#} \mathrm{P}<0.01$, compared with the control; ${ }^{*} \mathrm{P}<0.05$ and ${ }^{* *} \mathrm{P}<0.01$, compared with the LPS group, RTFs, flavonoids isolated from Radix Tetrastigmae; LPS, lipopolysaccharide; BALF, bronchoalveolar lavage fluid.

and TNF- $\alpha$ induced by LPS were markedly reduced by RTF treatment (Fig. 2A). Among the eight factors, BLC, GCSF and MIP-1 $\alpha$ belong to the family of chemotactic factors, which are predominantly involved in the infiltration of leukocytes. The downregulated expression levels of these factors were consistent with the numbers of leukocytes present in the BALF. These IL-1 $\beta$, IL- 6 , IL-12p40 and TNF- $\alpha$ proinflammatory factors may be the functional cytokines in LPS-induced ALI.

The release of IL-1 $\beta$, IL-6, IL-12p40 and TNF- $\alpha$ was also quantified using an ELISA assay. LPS treatment induced the release of cytokines to a maximum. The maximal induction for IL-6 was $220 \pm 11 \mathrm{pg} / \mathrm{ml}$, which was $\sim 5$-fold of that in the control. Compared with the model, the production of cytokines in the RTF-treated groups were significantly reduced in a dose-dependent manner (Fig. 2B). By comparing the results from the cytokine array analysis, the results from the ELISA assay revealed a more marginal change between groups, which may be accounted for by the sensitivity of the two different assay systems.

RTFs ameliorate histopathologic changes the in lung tissues of ALI. The severity of LPS-induced ALI was further evaluated. As shown in Fig. 3, the control group showed normal structures, and no histopathological changes were observed in the lung tissues. However, following challenge with LPS, the pulmonary function of the LPS group was markedly impaired, with various changes, including capillary congestion, hemorrhage, the infiltration or aggregation of neutrophils in airspace or vessel wall and thickening of the alveolar wall. In the experimental groups, the histopathological changes were abated by RTF treatment. In addition, similar inhibitory effects were exhibited in the semi-quantitative assay, in which histological changes were scored. The lung injury scores increased in the LPS-treated groups, however, the increase was significantly reduced by RTF treatment (Fig. 3; P<0.01). These results demonstrated that RTF treatment attenuated the severity of lung injury in the ALI mice induced by LPS, and improved the condition of the lung tissues. 


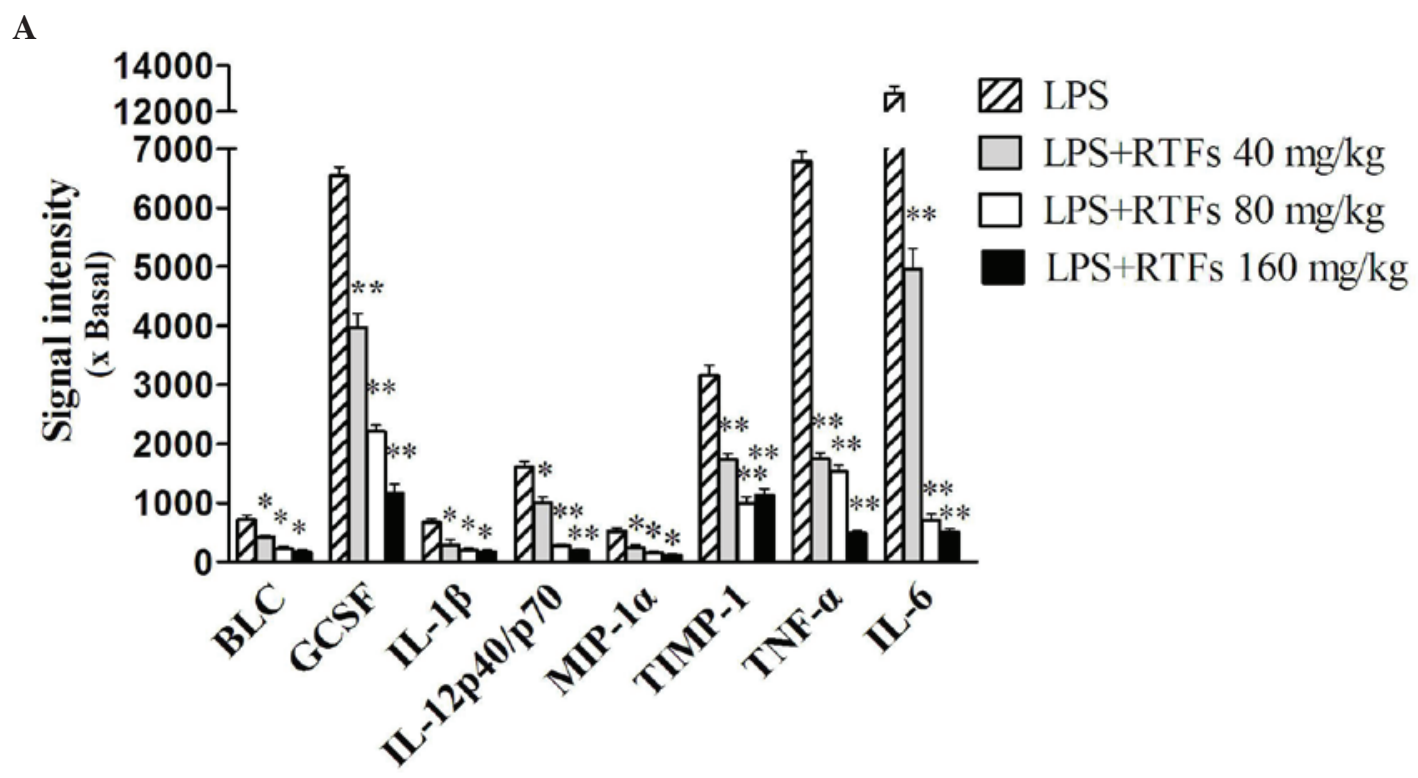

B

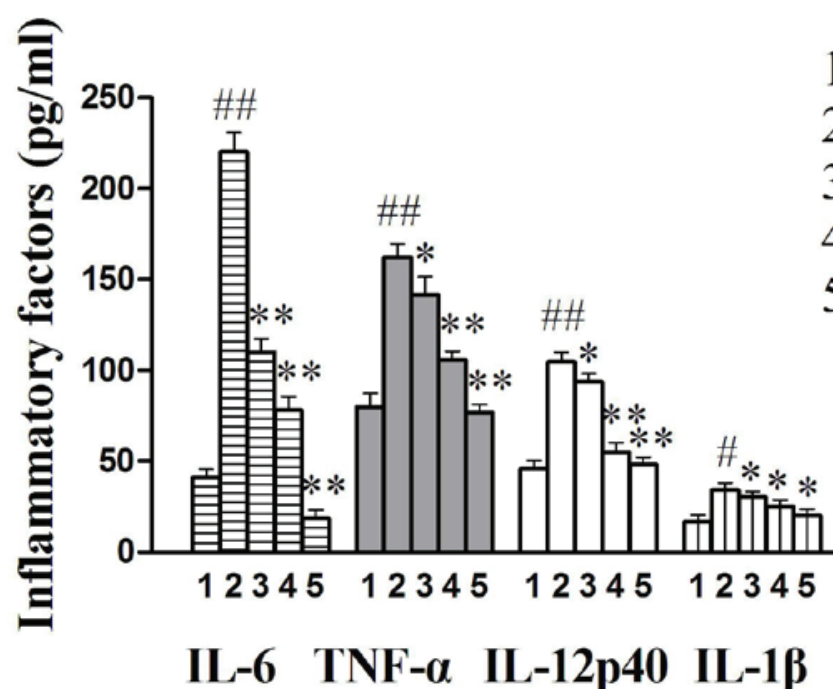

1:Control

2:LPS

3:LPS+RTFs $40 \mathrm{mg} / \mathrm{kg}$

4:LPS+RTFs $80 \mathrm{mg} / \mathrm{kg}$

5:LPS+RTFs $160 \mathrm{mg} / \mathrm{kg}$

Figure 2. Effect of RTFs on the secretion of inflammatory factors in bronchoalveolar lavage fluid from mice with LPS-induced ALI. (A) Following treatment with LPS and/or RTFs for 3 days, several inflammatory factors were found to be significantly altered in the inflammation antibody array. (B) Production of IL-1 $\beta$, IL-6, IL-12p40 and TNF- $\alpha$ was quantified using an ELISA assay, which showed significant reductions in a dose-dependent manner, compared with the LPS group. The data are expressed as the mean \pm standard deviation for each group. ${ }^{*} \mathrm{P}<0.05$ and ${ }^{\# \#} \mathrm{P}<0.01$, compared with the control; ${ }^{*} \mathrm{P}<0.05$ and ${ }^{* *} \mathrm{P}<0.01$, compared with the LPS group. RTFs, flavonoids isolated from Radix Tetrastigmae; LPS, lipopolysaccharide; BLC, B lymphocyte chemoattractant; GCSF, granulocyte colony stimulating factor; IL, interleukin; MIP-1 $\alpha$; macrophage inflammatory protein-1 $\alpha$ TIMP-1; tissue inhibitor of metalloproteinase-1; TNF- $\alpha$, tumor necrosis factor- $\alpha$.

RTFs inhibit the expression levels of TLR4 and MD-2 in lung tissues of ALI. To examine the mechanism underlying the anti-inflammatory effect of RTFs, the expression levels of TLR4 and MD-2 in the lung tissues were analyzed. LPS instillation markedly increased the protein levels of TLR4 and MD-2 in the mouse lungs (Fig. 4A), compared with the control. However, RTF treatment significantly inhibited the expression levels of TLR4 and MD-2 induced by LPS. As shown in Fig. 4B, the inhibitory effect was more marked on MD-2, compared with TLR4.

RTFs reduces $N F-\kappa B$ phosphorylation and DNA binding activity in lung tissues of $A L I$. NF- $\mathrm{KB}$ is an important upstream transcription factor, which induces the mRNA expression of various proinflammatory cytokines following stimulation with LPS. Western blot analysis (Fig. 4A and B) of the total protein extracts from the lung tissues showed that LPS treatment stimulated the expression and phosphorylation of NF- $\kappa \mathrm{B}$ p65 (Fig. 4C and D), which was also significantly downregulated by RTFs $(\mathrm{P}<0.05)$. Furthermore, the effect of RTF on the transactivation of NF-кB was also detected. As shown in Fig. 4E, RTFs significantly reduced the DNA binding activity of NF- $\mathrm{kB}$ in the nuclear extracts from lung tissues of the LPS-induced ALI mice, which occurred in a dose-dependent manner, as determined using the TransAM NF-kB kit $(\mathrm{P}<0.05)$.

RTFs alleviate MAPK activation in the lung tissues of ALI. The MAPK signaling pathway is another important pathway involved in inflammatory responses. Following 3 days of 


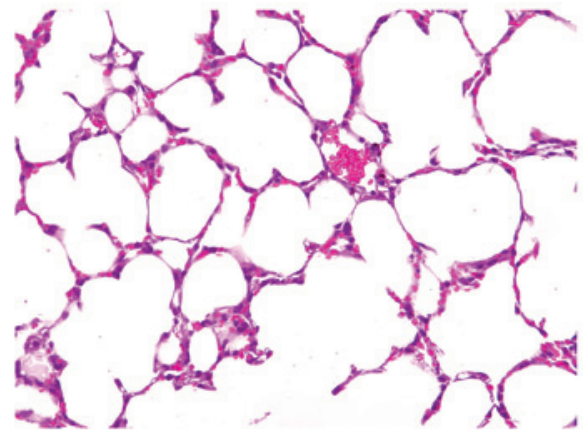

Control

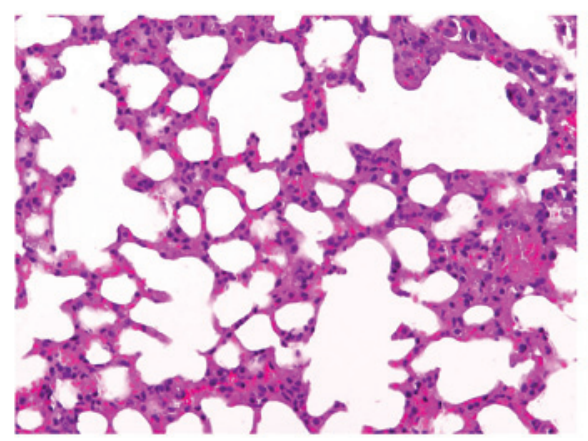

LPS+RTFs (40 mg/kg)

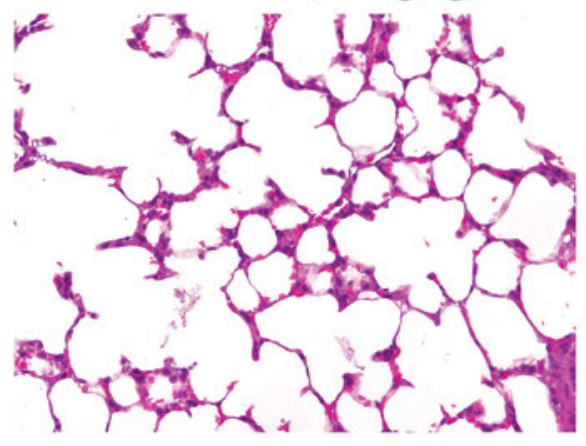

LPS+RTFs $(160 \mathrm{mg} / \mathrm{kg})$

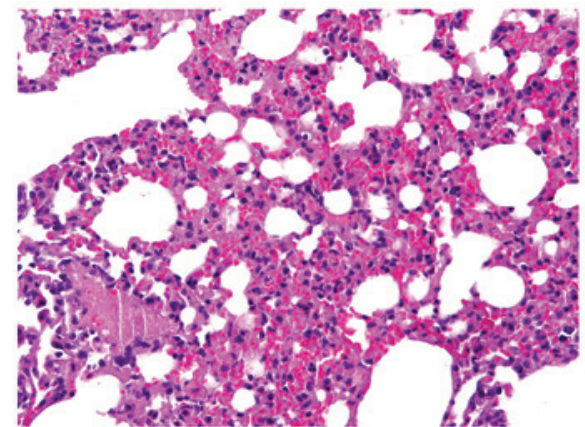

LPS

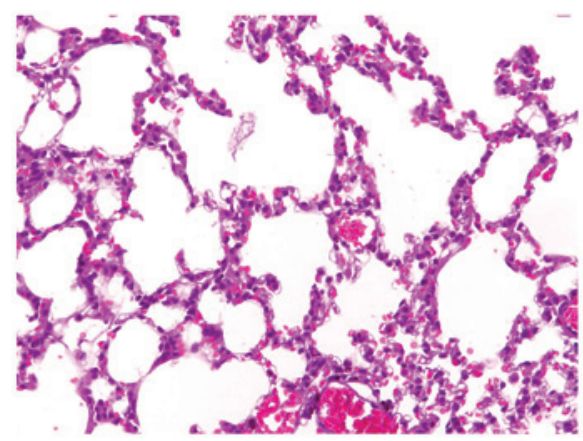

LPS+RTFs $(80 \mathrm{mg} / \mathrm{kg})$

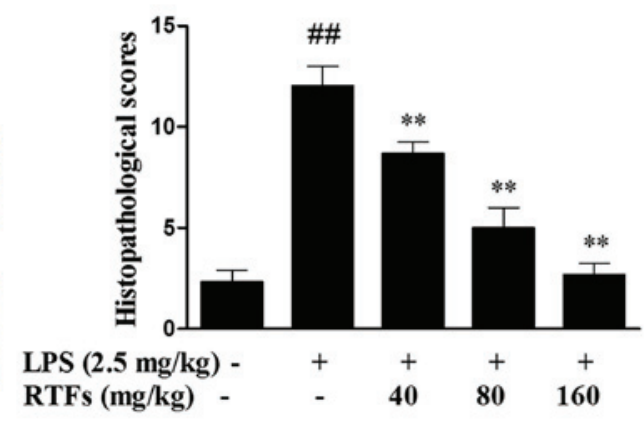

Figure 3. Effect of RTFs on LPS-induced histopathological changes in acute lung injury. Following treatment with LPS and/or RTFs for 3 days, the lung tissues were isolated. The effect of RTFs was assessed histologically in hematoxylin and eosin-stained sections and using histopathological scoring. Magnification, $\mathrm{x} 200$. Data are expressed as the mean \pm standard deviation for each group. ${ }^{\# \#} \mathrm{P}<0.01$, compared with the control; ${ }^{* *} \mathrm{P}<0.01$, compared with the LPS group. RTFs, flavonoids isolated from Radix Tetrastigmae; LPS, lipopolysaccharide.

LPS instillation, the MAPKs in the model group, including p38, JNK and ERK, showed markedly upregulated levels of expression and phosphorylation in the lungs, compared with the control group $(\mathrm{P}<0.01)$. When the LPS-induced ALI mice were treated with RTFs, the expression and phosphorylation levels of p38 and JNK were significantly reduced. ERK and p-ERK exhibited marginal differences between the groups, however, these differences were not statistically significant (Fig. 5).

\section{Discussion}

According to the theory of traditional Chinese medicine, RT tuberous roots can promote the production of body fluid and blood circulation, which results in improved lung ventilation. $\mathrm{RT}$ roots have anti-inflammatory and immunomodulatory activities, which can be used to treat bronchitis, pneumonia, pharyngolaryngitis and viral meningitis. RT significantly inhibits HAc-induced peritoneal capillary permeability, improves xylene-induced ear edema in mice and improves albumin-induced paw edema in rats (24). Feng et al (18) initially discussed the anti-inflammatory and antitoxic mechanisms from an immunological viewpoint; it was found that RT may be pivotal in the activation, proliferation and differentiation processes of $\mathrm{T}$ lymphocytes. Flavonoids are one of the predominant components in RT extract, therefore the present study aimed to elucidate the anti-inflammatory properties and mechanisms of RTF.

The recruitment of leukocytes is critical in the pathogenesis of ALI. It has been reported that neutrophil accumulation in the lung parenchyma is a histological hallmark of ALI (25). Leukocyte activation leads to the production of reactive oxygen species and granular enzymes, resulting in lung tissue injury by evoking an inflammatory cascade. In the present study, it was found that RTF significantly reduced the number of leukocytes in the BALF, including neutrophils and macrophages, 
A

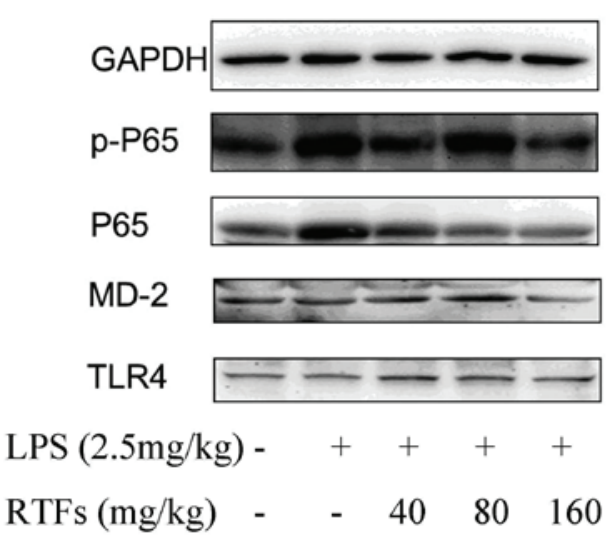

C

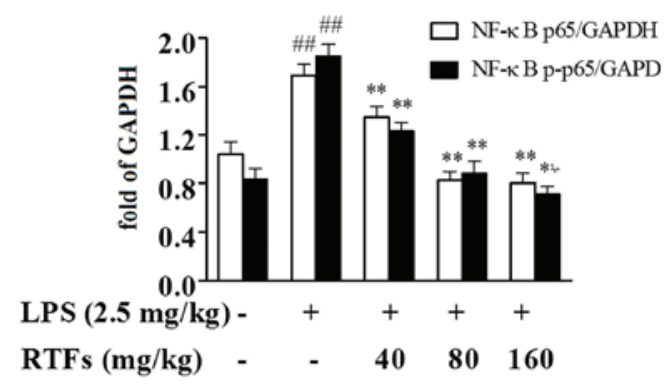

B

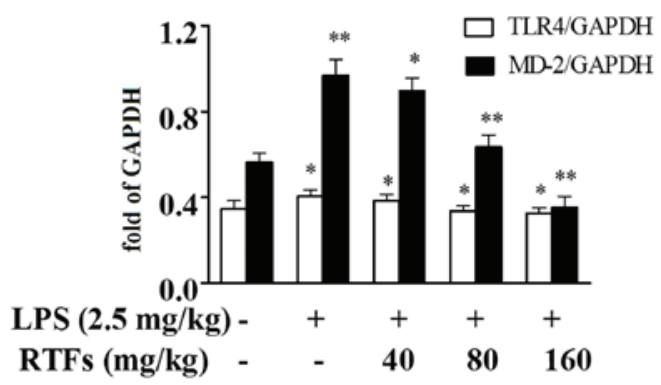

D

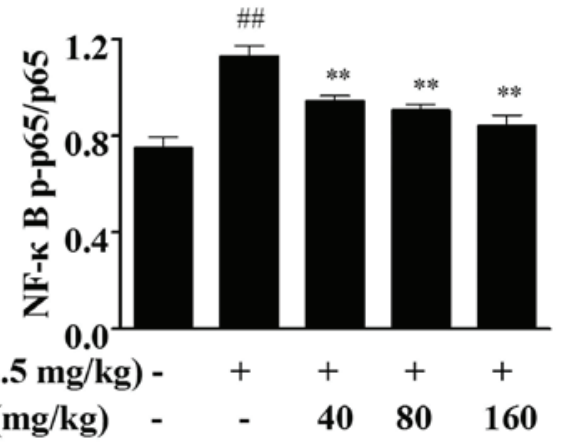

$\mathbf{E}$

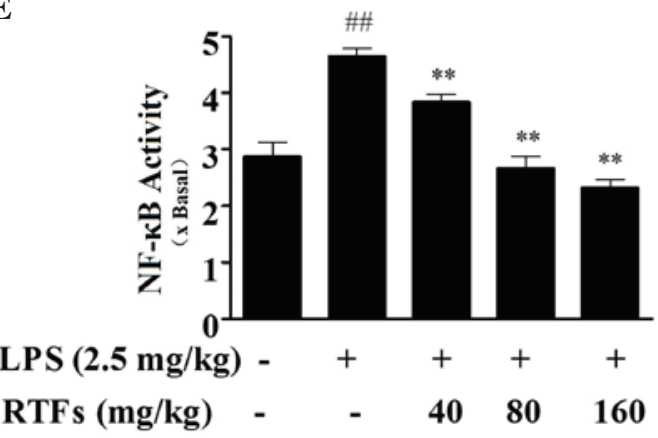

Figure 4. RTFs inhibit the TLR4/MD2-mediated NF- $\mathrm{B}$ signaling pathway in mice with LPS-induced ALI. (A) Protein expression levels of TLR4, MD-2 and $\mathrm{NF}-\kappa \mathrm{B}$ were examined in the lung homogenates 3 days following treatment with LPS and/or RTFs using western blot analysis. Increased expression levels of (B) TLR4 and MD-2, and (C) NF- $\kappa$ B were induced by LPS challenge. (D) Phosphorylation of NF- $\kappa$ B was significantly downregulated by RTF treatment. (E) DNA binding activity of NF- $\kappa \mathrm{B}$ in nuclear extracts from the lung tissues of the mice with LPS-induced ALI was attenuated by RTFs, determined using a TransAM NF- $\kappa$ B kit. The data are expressed as the mean \pm standard deviation for each group. ${ }^{\# \#} \mathrm{P}<0.01$, compared with the control; ${ }^{*} \mathrm{P}<0.05$ and ${ }^{* *} \mathrm{P}<0.01$, compared with the LPS group. RTFs, flavonoids isolated from Radix Tetrastigmae; LPS, lipopolysaccharide; ALI acute lung injury; TLR4, Toll-like receptor 4; MD-2, myeloid differentiation factor- 2 ; NF- $\kappa \mathrm{B}$, nuclear factor- $\kappa \mathrm{B} ; \mathrm{p}-$, phosphorylated.

following LPS challenge. In addition, RTFs significantly reduced macrophage infiltration in the BALF, which is consistent with the findings in our in vitro investigation, in which RTF treatment inhibited LPS-stimulated RAW264.7 monocyte activation (26). These results showed that RTFs effectively attenuated pulmonary inflammation in LPS-induced ALI.

It is widely accepted that inflammatory cytokines are crucial in the pathogenesis of LPS-induced ALI. Several cytokines, particularly IL- $1 \beta$, TNF- $\alpha$ and IL-6, have been identified as key factors in leukocyte infiltration in the lung tissues of ALI (27). In the present study, an inflammation microarray was used, which detected marked increases in the levels of a series of inflammatory mediators in the lung tissues following LPS instillation, including the chemotatic factors, GCSF, MIP-1 $\alpha$ and BLC, and inflammatory cytokines, IL-1 $\beta$, TNF- $\alpha$, IL- 6 and IL-12p40. However, the secretion of these mediators were all significantly reduced with RTF treatment. The change in chemotactic factors is consistent with the leukocyte infiltration in the BALF of the ALI mice. The production of inflammatory cytokines was further confirmed using an ELISA assay. The findings confirmed that the protective effect of RTFs on LPS-induced ALI may be attributed to the inhibition of pulmonary inflammatory responses.

Previous studies have indicated that TLR4 is the major signaling receptor in LPS-induced inflammatory 

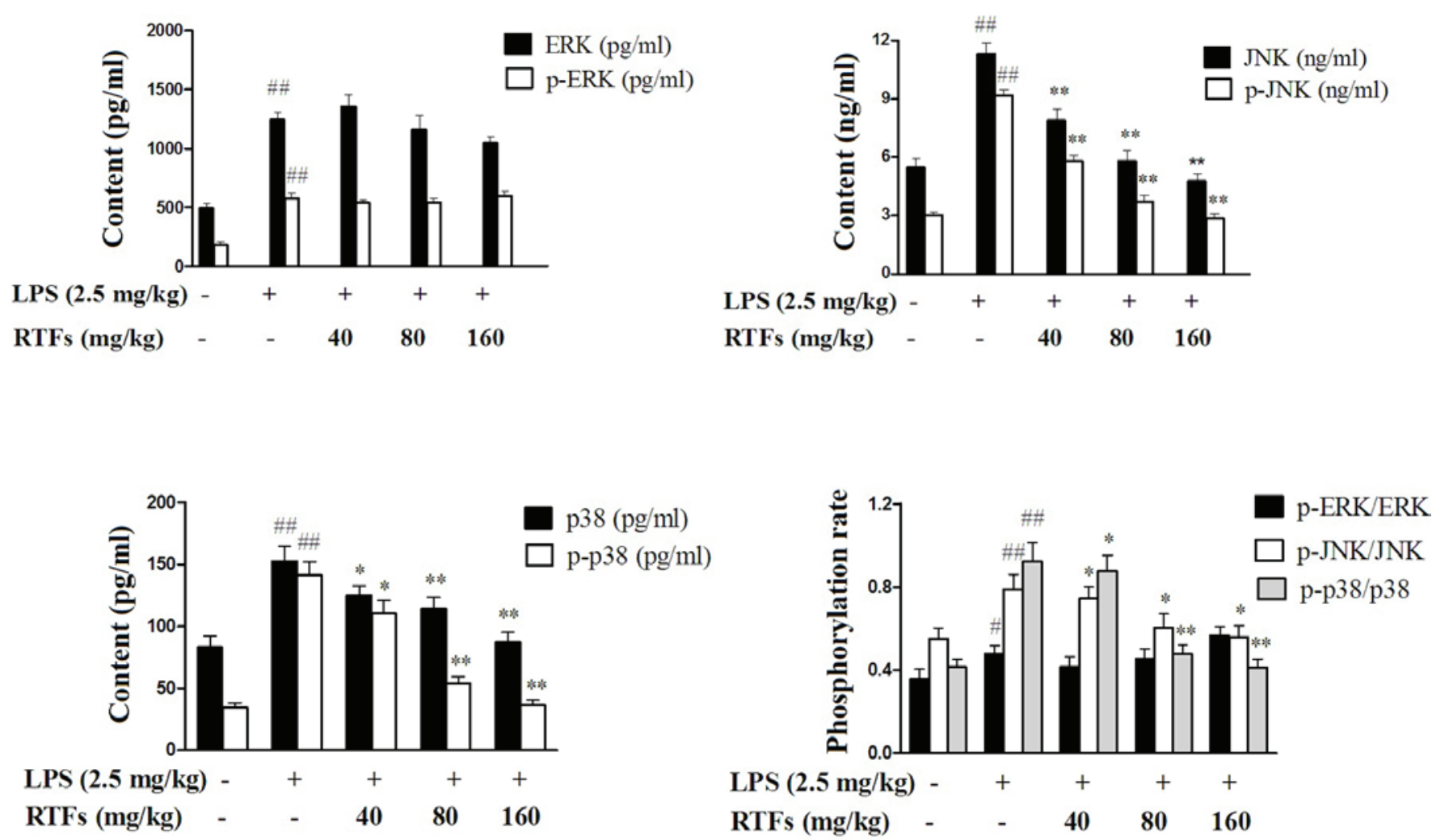

Figure 5. RTFs inhibit the mitogen-activated protein kinase signaling pathway in lung tissues of mice with LPS-induced acute lung injury. Following treatment with LPS and/or RTFs for 3 days, the protein expression levels of JNK, p38, ERK and their phosphorylated forms were examinedin the lung homogenates using ELISA. JNK, p38 and their phosphorylated forms showed significantly downregulated levels of expression and phosphorylation in the RTF-treated groups. However, ERK and p-ERK exhibited only marginal differences. The data are expressed as the mean \pm standard deviation of $10-12$ mice/group., ${ }^{\#} \mathrm{P}<0.05$ and ${ }^{\# \#} \mathrm{P}<0.01$, compared with the control; ${ }^{*} \mathrm{P}<0.05$ and ${ }^{* *} \mathrm{P}<0.01$, compared with the LPS group. RTFs, flavonoids isolated from Radix Tetrastigmae; LPS, lipopolysaccharide; JNK, c-Jun N-terminal kinase; ERK, extracellular signal-regulated kinase; p-, phosphorylated.

responses (28) by regulating the activities of downstream mediators, including MAPKs and NF- $\mathrm{KB}(29,30)$. MD-2, which has been shown to be essential for the correct intracellular distribution of TLR4 and efficient LPS recognition, mediates LPS-induced responses in vitro and in vivo (31-33). Accumulating evidence has shown that MD-2 is the critical receptor protein for LPS and is necessary for the ligand-dependent dimerization of the TLR4/MD-2 complex (31-33). The binding of the TLR4/MD-2 complex receptor by microbial products triggers a downstream signaling cascade, culminating in the activation of $\mathrm{NF}-\kappa \mathrm{B}$, and promoter recognition sites direct the production of a number of pro-inflammatory cytokines, including IL-1 $\beta, \mathrm{TNF}-\alpha$ and IL-6 (34). In the present study, RTFs inhibited the expression of MD-2 and TLR4. Although the effect was more marked on MD-2, compared with TLR4, they synergistically led to a reduction in TLR4/MD-2 complex formation. These data suggested that RTFs may attenuate the severity of LPS-induced local inflammation, activated through the TLR4/MD-2-mediated signaling pathway.

Activation of the TLR4/MD-2 pathway initiates a series of MAPK and NF- $\kappa \mathrm{B}$ cascades, and results in the overproduction of cytokines, including TNF- $\alpha$ and IL-6 (35). $\mathrm{NF}-\kappa \mathrm{B}$ is an important transcription factor in the LPS-TLR4 signaling pathway, regulating immune and inflammatory processes $(36,37)$. The activation of MAPKs is critical in mediating a broad array of cellular responses, including cell proliferation and differentiation, transcription factor activation, and cytokine gene expression and production (38-40). Thus, inhibitors of NF- $\mathrm{KB}$ and MAPKs have been used as therapeutic drugs in clinical applications for inflammation-associated human diseases $(41,42)$. In the present study, it was shown that RTFs potently suppressed LPS-induced NF- $\kappa$ B p65 activation. In addition to decreases in the expression and phosphorylation of $\mathrm{NF}-\kappa \mathrm{B}$ p 65 in the total protein extracted from lung tissues, a reduction in DNA binding activity of intranuclear NF- $\mathrm{NB}$ p65 was also observed following RTFs treatment, suggesting that RTFs inhibited the mobilization of p65 into the nucleus and attenuated NF- $\mathrm{B}$-mediated signaling transduction. In addition, RTFs inhibited LPS-induced JNK and p38MAPK phosphorylation. Inhibition of the activation of JNK, p38MAPK and $N F-\kappa B$ result in decreases in the expression levels of cytokines, including IL-1 $\beta$, IL-6, TNF- $\alpha$ and IL-12 (43). Thus, the inhibitory property of RTFs on LPS-induced generation of proinflammatory cytokines may be associated with the attenuated activation of JNK, p38MAPK and $\mathrm{NF}-\kappa \mathrm{B}$.

The present study was the first, to the best of our knowledge, to demonstrate that total flavonoids isolated from RT significantly decreased the production of proinflammatory factors in an LPS-induced ALI mouse model. It was found that RTFs may interact with the expression and formation of the 
TLR4/MD-2 complex to inhibit the action of LPS. RTF treatment protected the lung tissues of the ALI mice from excessive phosphorylation of JNK, p38MAPK and NF- $\mathrm{kB}$, and downregulated the expression and DNA-binding activity of NF- $\kappa B$ through the TLR4/MD-2 pathway. Collectively, the results of the present study provide novel insights into the mechanisms of RTFs as anti-inflammatory agents for LPS-mediated infections, which may be a potential therapeutic agent for septic shock.

\section{Acknowledgements}

The authors would like to thank Dr Weihong Ge for their expert guidance and support, and would like to thank the members of the pharmacological team and research department of Zhejiang Medical College for their assistance. This study was supported by the grant of Zhejiang Modernization of Traditional Chinese Medicine (grant no. 2010350).

\section{References}

1. Gotts JE and Matthay MA: Treating ARDS: New hope for a tough problem. Lancet Respir Med 2: 84-85, 2014.

2. Fernandez-Bustamante A and Repine JE: Chronic inflammatory diseases and the acute respiratory distress syndrome (ARDS). Curr Pharm Des 20: 1400-1408, 2014.

3. Ware LB: Autopsy in ARDS: Insights into natural history. Lancet Respir Med 1: 352-354, 2013.

4. Thompson BT and Matthay MA. The Berlin definition of ARDS versus pathological evidence of diffuse alveolar damage. Am J Respir Crit Care Med 187: 675-677, 2013.

5. De Luca D, Piastra M, Tosi F, Pulitanò S, Mancino A, Genovese O, Pietrini D and Conti G: Pharmacological therapies for pediatric and neonatal ALI/ARDS: An evidence-based review. Curr Drug Targets 13: 906-916, 2012.

6. Zhu T, Wang DX, Zhang W, Liao XQ, Guan X, Bo H, Sun JY, Huang NW, He J, Zhang YK, et al: Andrographolide protects against LPS-induced acute lung injury by inactivation of NF- $\kappa$. PLoS One 8: e56407, 2013.

7. Matute-Bello G, Frevert CW and Martin TR: Animal models of acute lung injury. Am J Physiol Lung Cell Mol Physiol 295: L379-L399, 2008.

8. Beduschi MG, Guimarães CL, Buss ZS and Dalmarco EM: Mycophenolate mofetil has potent anti-inflammatory actions in a mouse model of acute lung injury. Inflammation 36: 729-737, 2013.

9. Song DH and Lee JO: Sensing of microbial molecular patterns by Toll-like receptors. Immunol Rev 250: 216-229, 2012.

10. Kim HJ, Lee HS, Chong YH and Kang JL: p38 mitogen-activated protein kinase up-regulates LPS-induced NF-kappaB activation in the development of lung injury and RAW 264.7 macrophages. Toxicology 225: 36-47, 2006

11. Arndt PG, Young SK, Lieber JG, Fessler MB, Nick JA and Worthen GS: Inhibition of c-Jun N-terminal kinase limits lipopolysaccharide-induced pulmonary neutrophil influx. Am J Respir Crit Care Med 171: 978-986, 2005.

12. Xiao M,Zhu T, Wang T and Wen FQ: Hydrogen-rich saline reduces airway remodeling via inactivation of NF- $\kappa \mathrm{B}$ in a murine model of asthma. Eur Rev Med Pharmacol Sci 17: 1033-1043, 2013.

13. Zhu T, Zhang W, Xiao M, Chen $\mathrm{H}$ and Jin H: Protective role of andrographolide in bleomycin-induced pulmonary fibrosis in mice. Int J Mol Sci 14: 23581-23596, 2013.

14. Wang X, Zhang L, Duan W, Liu B, Gong P, Ding Y and Wu X: Anti-inflammatory effects of triptolide by inhibiting the NF- $\kappa \mathrm{B}$ signalling pathway in LPS-induced acute lung injury in a murine model. Mol Med Rep 10: 447-452, 2014.

15. Jin LY, Li CF, Zhu GF, Wu CT, Wang J and Yan SF: Effect of siRNA against NF- $\kappa B$ on sepsis-induced acute lung injury in a mouse model. Mol Med Rep 10: 631-637, 2014.

16. Smyth K, Garcia K, Sun Z, Tuo W and Xiao Z: TLR agonists are highly effective at eliciting functional memory CTLs of effector memory phenotype in peptide immunization. Int Immunopharmacol 15: 67-72, 2013.
17. Chen LY and Guo SH: Progress in studies of chemical composition and pharmacological effects of Tetrastigmatis Hems Leyani. Zhejiang Zhong Yi Xue Yuan Xue Bao 12: 1368-1370, 2012.

18. Feng Z, Hao W, Lin X, Fan D and Zhou J: Antitumor activity of total flavonoids from Tetrastigma hemsleyanum Diels et Gilg is associated with the inhibition of regulatory $\mathrm{T}$ cells in mice. Onco Targets Ther 7: 947-956, 2014.

19. Ma D, Li W, Ma Z, et al: Anti-liver damage activity analysis of polysaccharide in Radix Tetrastigmatis Hemsleyani. J Med Res 41: 33-36, 2012.

20. Liu B, Yang J, Ma Y, Yuan E and Chen C: Antioxidant and angiotensin converting enzyme (ACE) inhibitory activities of ethanol extract and pure flavonoids from Adinandra nitida leaves. Pharm Biol 48: 1432-1438, 2010.

21. Chen L, Ding L, Yu A, Yang R, Wang X, Li J, Jin H and Zhang H: Continuous determination of total flavonoids in Platycladus orientalis (L.) Franco by dynamic microwave-assisted extraction coupled with on-line derivatization and ultraviolet-visible detection. Anal Chim Acta 596: 164-170, 2007.

22. Guo L, Li WJ, Xu MJ and Wang X: A mouse model of acute lung inflammation induced by lipopolysaccharide inhalation. Beijing Da Xue Xue Bao 41: 226-229, 2009 (In Chinese).

23. Chiu CJ, McArdle AH, Brown R, Scott HJ and Gurd FN: Intestinal mucosal lesion in low-flow states. I. A morphological, hemodynamic and metabolic reappraisal. Arch Surg 101: 478-483 1970.

24. Huang Z, Mao Q and Wei J: Evaluation of anti-inflammatory, analgesic and antipyretic actions for the extracts from Radix Tetrastigmae. Chinese Journal of New Drugs 14: 861-864, 2005.

25. Zhou X, Dai Q and Huang X: Neutrophils in acute lung injury. Front Biosci (Landmark Ed) 17: 2278-2283, 2012.

26. Liu D, Cao G, Han L, Ye Y, SiMa Y and Ge W: Flavonoids from Radix Tetrastigmae inhibit TLR4/MD-2 mediated JNK and NF- $\kappa B$ pathway with anti-inflammatory properties. Cytokine 84: 29-36, 2016.

27. Zhang H, Neuhöfer P, Song L, Rabe B, Lesina M, Kurkowski MU, Treiber M, Wartmann T, Regnér S, Thorlacius H, et al: IL-6 trans-signaling promotes pancreatitis-associated lung injury and lethality. J Clin Invest 123: 1019-1031, 2013.

28. Gioannini TL, Teghanemt A, Zhang D, Coussens NP, Dockstader W, Ramaswamy S and Weiss JP: Isolation of an endotoxin-MD-2 complex that produces Toll-like receptor 4-dependent cell activation at picomolar concentrations. Proc Natl Acad Sci USA 101: 4186-4191, 2004.

29. Baker RG, Hayden MS and Ghosh S: NF- $\mathrm{BB}$, inflammation, and metabolic disease. Cell Metab 13: 11-22, 2011.

30. Theoharides TC, Kempuraj D, Tagen M, Conti $\mathrm{P}$ and Kalogeromitros D: Differential release of mast cell mediators and the pathogenesis of inflammation. Immunol Rev 217: 65-78, 2007.

31. Wolfs TG, Dunn-Siegrist I, van't Veer C, Hodin CM, Germeraad WT, van Zoelen MA, van Suylen RJ, Peutz-Kootstra CJ, Elson G, Pugin J and Buurman WA: Increased release of sMD-2 during human endotoxemia and sepsis: A role for endothelial cells. Mol Immunol 45: 3268-3277, 2008.

32. Schnabl B, Brandl K, Fink M, Gross P, Taura K, Gäbele E, Hellerbrand C and Falk W: A TLR4/MD2 fusion protein inhibits LPS-induced pro-inflammatory signaling in hepatic stellate cells. Biochem Biophys Res Commun 375: 210-214, 2008.

33. Zhang J, Kumar A, Wheater M and Yu FS: Lack of MD-2 expression in human corneal epithelial cells is an underlying mechanism of lipopolysaccharide (LPS) unresponsiveness. Immunol Cell Biol 87: 141-148, 2009.

34. Akira S, Uematsu S and Takeuchi O: Pathogen recognition and innate immunity. Cell 124: 783-801, 2006.

35. Xie G, Chen N, Soromou LW, Liu F, Xiong Y, Wu Q, Li H, Feng H and Liu G: p-Cymene protects mice against lipopolysaccharide-induced acute lung injury by inhibiting inflammatory cell activation. Molecules 17: 8159-8173, 2012.

36. Ling M, Li Y, Xu Y, Pang Y, Shen L, Jiang R, Zhao Y, Yang X, Zhang J, Zhou J, et al: Regulation of miRNA-21 by reactive oxygen species-activated ERK/NF- $\kappa \mathrm{B}$ in arsenite-induced cell transformation. Free Radic Biol Med 52: 1508-1518, 2012.

37. Han DW, Lee MH, Kim HH, Hyon SH and Park JC: Epigallocatechin-3-gallate regulates cell growth, cell cycle and phosphorylated nuclear factor- $\kappa \mathrm{B}$ in human dermal fibroblasts. Acta Pharmacol Sin 32: 637-646, 2011. 
38. Johnson GL and Lapadat R: Mitogen-activated protein kinase pathways mediated by ERK, JNK, and p38 protein kinases Science 298: 1911-1912, 2002.

39. Dai JN, Zong Y, Zhong LM, Li YM, Zhang W, Bian LG, Ai QL, Liu YD, Sun J and Lu D: Gastrodin inhibits expression of inducible NO synthase, cyclooxygenase-2 and proinflammatory cytokines in cultured LPS-stimulated microglia via MAPK pathways. PloS One 6: e21891, 2011.

40. Liu HT, Huang P, Ma P, Liu QS, Yu C and Du YG: Chitosan oligosaccharides suppress LPS-induced IL-8 expression in human umbilical vein endothelial cells through blockade of p38 and Akt protein kinases. Acta Pharmacol Sin 32: 478-486, 2011.
41. Liu C, Zhang X, Zhou JX, Wei W, Liu DH, Ke P, Zhang GF, Cai GJ and Su DF: The protective action of ketanserin against lipopolysaccharide-induced shock in mice is mediated by inhibiting inducible NO synthase expression via the MEK/ERK pathway. Free Radic Biol Med 65: 658-666, 2013.

42. Choi Y, Lee MK, Lim SY, Sung SH and Kim YC: Inhibition of inducible NO synthase, cyclooxygenase-2 and interleukin-1beta by torilin is mediated by mitogen-activated protein kinases in microglial BV2 cells. Br J Pharmacol 156: 933-940, 2009.

43. Xu X, Yin P, Wan C, Chong X, Liu M, Cheng P, Chen J, Liu F and $\mathrm{Xu}$ J: Punicalagin inhibits inflammation in LPS-induced RAW264.7 macrophages via the suppression of TLR4-mediated MAPKs and NF-кB activation. Inflammation 37: 956-965, 2014. 